\title{
Morphological changes in diabetic kidney are associated with increased $O$-GlcNAcylation of cytoskeletal proteins including $\alpha$-actinin 4
}

Yoshihiro Akimoto ${ }^{1 *}$, Yuri Miura ${ }^{2}$, Tosifusa Toda ${ }^{2}$, Margreet A Wolfert ${ }^{3}$, Lance Wells $s^{3,4,5}$, Geert-Jan Boons ${ }^{3,4}$, Gerald W Hart ${ }^{6}$, Tamao Endo ${ }^{2}$ and Hayato Kawakami ${ }^{1}$

* Correspondence: yakimoto@ks. kyorin-u.ac.jp

'Department of Anatomy, Kyorin University School of Medicine, Mitaka, Tokyo 181-8611, Japan Full list of author information is available at the end of the article

\begin{abstract}
Purpose: The objective of the present study is to identify proteins that change in the extent of the modification with O-linked $\mathrm{N}$-acetylglucosamine (O-GlcNAcylation) in the kidney from diabetic model Goto-Kakizaki (GK) rats, and to discuss the relation between O-GlcNAcylation and the pathological condition in diabetes.

Methods: O-GIcNAcylated proteins were identified by two-dimensional gel electrophoresis, immunoblotting and peptide mass fingerprinting. The level of O-GlcNAcylation of these proteins was examined by immunoprecipitation, immunoblotting and in situ Proximity Ligation Assay (PLA).

Results: $O-G$ lcNAcylated proteins that changed significantly in the degree of $O$-GlcNAcylation were identified as cytoskeletal proteins ( $\alpha$-actin, $\alpha$-tubulin, $\alpha$-actinin 4 , myosin) and mitochondrial proteins (ATP synthase $\beta$, pyruvate carboxylase). The extent of O-GlcNAcylation of the above proteins increased in the diabetic kidney.

Immunofluorescence and in situ PLA studies revealed that the levels of O-GlcNAcylation of actin, $\alpha$-actinin 4 and myosin were significantly increased in the glomerulus and the proximal tubule of the diabetic kidney. Immunoelectron microscopy revealed that immunolabeling of $\alpha$-actinin 4 is disturbed and increased in the foot process of podocytes of glomerulus and in the microvilli of proximal tubules.

Conclusion: These results suggest that changes in the O-GlcNAcylation of cytoskeletal proteins are closely associated with the morphological changes in the podocyte foot processes in the glomerulus and in microvilli of proximal tubules in the diabetic kidney. This is the first report to show that $\alpha$-actinin 4 is O-GlcNAcylated. $\alpha$-Actinin 4 will be a good marker protein to examine the relation between 0 GlcNAcylation and diabetic nephropathy.
\end{abstract}

Keywords: O-GlcNAc modification, Hexosamine biosynthetic pathway, Kidney, Glomerulus, Cytoskeleton, $\alpha$ ? a?-actinin, GK Rat, Mass spectrometry, Proximity Ligation Assay

\section{Introduction}

$O$-linked $N$-acetyl- $\beta$ - $D$-glucosamine, termed $O$-GlcNAc, is a post-translational modification involved in modulation of signaling and transcription in response to cellular nutrients or stress by interplay with $O$-phosphorylation [1-3]. O-GlcNAc serves as a glucose sensor via the hexosamine biosynthetic pathway. Elevated O-GlcNAc modification (O-GlcNAcylation) of proteins by increased flux through the hexosamine

\section{() Biomed Central}


biosynthetic pathway has been implicated in the development of insulin resistance and diabetic complications and in the up-regulated gene expression of transforming growth factor-beta1, plasminogen activator inhibitor 1, and upstream stimulatory factor proteins in mesangial cells, leading to mesangial matrix expansion and diabetic glomerulosclerosis [2,4-9]. We previously demonstrated increased O-GlcNAcylation in the kidney and pancreas of the Goto-Kakizaki (GK) rat, which is an animal model of type 2 diabetes $[10,11]$. Also, altered $O$-GlcNAcylation and $O$-GlcNAc transferase (OGT) expression were recently reported in the kidney from diabetic patients [12].

In this study we carried out proteomic analysis, especially focused on the proteins with remarkable change of the $O$-GlcNAc level in the kidney from GK rats, and suggested the potential of $O$-GlcNAcylation as a biomarker of diabetic nephropathy. Total kidney proteins from Wistar and GK rats were separated by two-dimensional gel electrophoresis. $O$-GlcNAcylated proteins were detected by immunoblotting using anti-O-GlcNAc antibody. Selected proteins that changed markedly in their extent of $O$-GlcNAcylation were identified by Mass Spectrometry (MS) analysis. MS sequencing of tryptic peptides identified some cytoskeletal proteins, including $\alpha$-tubulin and $\alpha$-actinin 4 . Immunoprecipitation and immunoblot findings demonstrated that $O$-GlcNAcylation of these identified proteins was increased in the diabetic rats. To examine the localization of the identified cytoskeletal proteins, we conducted an immunohistochemical study using confocal scanning microscopy and immuno-electron microscopy. The localization and quantity of these O-GlcNAcylated proteins were further examined by performing the in situ Proximity Ligation Assay (PLA), which was developed to examine protein-to-protein interaction and post-translational modification of proteins $[13,14]$.

\section{Methods}

\section{Animals and tissues}

Kidney tissues were obtained by dissecting 15 -week-old male $(\mathrm{n}=3)$ Wistar rats (as controls) and GK rats, which are a nonobese model of non-insulin-dependent diabetes mellitus and had been developed by the selective breading of glucose-intolerant Wistar rats. Both rats were obtained from CLEA (Tokyo, Japan). All experimental procedures using laboratory animals were approved by the Animal Care and Use Committee of Kyorin University School of Medicine.

\section{Reagents}

Rabbit polyclonal anti- $\alpha$-actinin 4 antibody was obtained from LifeSpan BioSciences (Seattle, WA). Rabbit polyclonal anti-myosin antibody was obtained from Biomedical Technologies (Stoughton, MA). Rabbit monoclonal anti-actin antibody (clone EP184E) and rabbit monoclonal anti- $\alpha$-tubulin antibody (clone EP1332Y) were obtained from Epitomics (Burlingame, CA). Mouse monoclonal anti-O-GlcNAc antibodies (CTD110.6, 18B10.C7 [3]) were used. The generation of CTD110.6, 18B10.C7(3) was previously described $[15,16]$.

\section{Two-dimensional gel electrophoresis (2D-PAGE) and immunoblotting}

Protein extraction and 2D-PAGE were performed as previously reported [17-19]. Three nondiabetic and 3 diabetic rat kidneys were used simultaneously from protein extraction to gel matching. Five-hundred micrograms of total protein prepared from normal 
and diabetic kidneys was loaded onto the gel for isoelectric focusing, which was performed by using pre-cast immobilized $\mathrm{pH}$ gradient (IPG) strips $(18 \mathrm{~cm}$ long, $\mathrm{pH} 4-7$, GE Healthcare Science). After equilibration in reducing solution and then in alkylating solution, second-dimensional gel electrophoresis was performed by $10 \%$ SDS-PAGE. Separated protein spots on polyacrylamide gels were electroblotted onto PVDF membranes. Then total protein spots were stained with BODIPY FL-X (Invitrogen). The membranes were first blocked for $1 \mathrm{~h}$ at room temperature with $0.3 \%$ BSA in TBS-T and then incubated with mouse monoclonal anti-O-GlcNAc antibody (CTD 110.6, Covance) at a dilution of 1:5,000 for $1 \mathrm{hr}$ at room temperature, followed by incubation with biotin-goat anti mouse IgM (Vector) at a dilution of 1:2,000 and then Qdot 625conjugated streptavidin (Invitrogen) at a dilution of 1:2,000, each for $1 \mathrm{hr}$ at room temperature. The total spots and immunoreactive spots were scanned by using a Molecular Imager FX laser scanning fluorometer (BioRad Laboratories, Hercules, CA). The intensities of spots were analyzed by using PDQuest software ver.8.0 (BioRad Lab). The search for protein spots whose O-GlcNAcylation had changed in the GK sample was performed as described previously [17]. The abundance of spots was presented as parts per million of the total spots integrated by using the 'total quantity in analysis set' feature of the PDQuest software. When the abundance of spots on 2D gels of diabetic kidneys was $>2$-fold or less than 0.5-fold compared with that for the control nondiabetic kidneys, we regarded the spots as proteins with a changed $O$-GlcNAcylation level.

\section{In-gel protein digestion and peptide mass fingerprinting}

The selected spots were cut from the second dimensional gel stained with SYPRORuby. In-gel protein digestion of selected gel spots was performed according to the protocol described http://www.proteome.jp/2D/2DE_method.html[18]. Peptide-mass fingerprinting data were acquired by using a MALDI-TOF-MS spectrometer (AXIMACFR, Shimazu Biotech). Proteins were identified with the Mascot search engine (Matrix Science, London, UK) search algorithms by using the Swiss-Prot protein databases (Version 57.6).

Immunoprecipitation, immuno-blotting and immunohistochemical study of actin, $\alpha$ actinin $4, \alpha$-tubulin, and myosin

Immunoprecipitation, immuno-blot analysis and immunohistochemical study were carried out as described previously [11].

\section{In situ PLA analysis}

In situ PLA analysis was performed according to the manufacturer's instructions with an HRP/NovaRed detection kit from Olink Bioscience (Uppsala, Sweden) [20]. Cryostat sections of kidney were cut and placed onto MAS-coated slides (Matsunami Glass, Tokyo, Japan). The slides were then incubated at $60^{\circ} \mathrm{C}$ in LAB solution for $5 \mathrm{~min}$. These antigen-retrieved tissues were next washed with PBS, incubated for $15 \mathrm{~min}$ in $15 \mathrm{ml} / \mathrm{L}$ hydrogen peroxide in PBS, washed, and blocked with 1\% BSA-PBS. For the in situ ligation assay, we used mouse monoclonal anti-O-GlcNAc antibodies (clone: 18B10.C7 [3]). The sections were incubated with this mouse anti-O-GlcNAc antibody in combination with rabbit anti-actin, anti- $\alpha$-actinin 4 , anti- $\alpha$-tubulin or anti-myosin 
antibody overnight at $4^{\circ} \mathrm{C}$. After having been washed with TBS-0.1\% Tween20 (TBS-T), the sections were incubated with a mixture of MINUS secondary PLA probe against mouse immunoglobulins and PLUS secondary PLA probe against rabbit immunoglobulins for $1 \mathrm{~h}$ at $37^{\circ} \mathrm{C}$. Then they were washed with TBS-T, and subsequently incubated in hybridization solution for $15 \mathrm{~min}$ at $37^{\circ} \mathrm{C}$ and washed with TBS-T once for $1 \mathrm{~min}$. The slides were next incubated with the ligation mix for $30 \mathrm{~min}$ at $37^{\circ} \mathrm{C}$ and washed with TBS-T twice for $1 \mathrm{~min}$ each time. Then the sections were incubated with the amplification mix for $90 \mathrm{~min}$ at $37^{\circ} \mathrm{C}$ and washed 3 times for 5 min each time with TBS-T. The slides were thereafter incubated with the HRP-labeled hybridization probe for $30 \mathrm{~min}$ at room temperature. After 2 washes with TBS for 2 min each time, the slides were incubated with DAB staining mix for 5 min and then washed with water. Nuclei were stained with hematoxyline. The slides were examined with a bright-field microscope equipped with a CCD camera Pro600ES (Pixera, San Jose, CA). In the negative controls in which the primary antibodies had been replaced with normal rabbit IgG and normal mouse IgG or omitted, signals were scarcely observed (data not shown). Signals were quantified with BlobFinderBright software, which is available on BlobFinder Website http://www.cb.uu.se/ amin/BlobFinder/[20].

\section{Statistics}

Data were compiled from 3 independent experiments. Student's t-test was used for statistical analysis, and for all cases $\mathrm{P}<0.05$ was considered to indicate statistical significance.

\section{Results}

\section{Identification of O-GlcNAcylated proteins in normal and diabetic kidneys}

To identify $O$-GlcNAcylated proteins, we employed two-dimensional electrophoresis and immunoblotting using the CTD110.6 anti-O-GlcNAc antibody. Total proteins of diabetic GK rat kidney or nondiabetic Wistar rat kidney were electrophoresed on a two-dimensional gel. Approximately 1,000 protein spots were detected in each SYPRO Ruby-stained gel (Figure 1A, B). Approximately 100 protein spots were detected in each Qdot 625-stained PVDF membrane (Figure 1C, D). The immunofluorescence intensity of each spot was quantified and compared between the nondiabetic kidney and the diabetic one. This comparison revealed enhanced $O$-GlcNAcylation in many proteins from the diabetic kidney (Figure 1C-P). Twenty-seven spots that indicated a significant difference in the relative quantity of O-GlcNAcylated protein were applied to MALDI-TOF MS for identification of the proteins. The identified proteins included various cytoskeletal proteins ( $\alpha$-tubulin, $\alpha$-actinin 4 , myosin, actin) and mitochondrial proteins (ATP synthase $\beta$ pyruvate carboxylase), which were temporarily referred to as spots "a"-"f" (Figure 1, Table 1). The intensity of these spots increased in the diabetic kidneys (Table 1). Almost the same results were obtained among the 3 GK rats as well as the 3 control rats. These proteins except for $\alpha$-actinin 4 have already been reported to be $O$-GlcNAcylated.

In the next step we focused on the cytoskeletal proteins, as they play important roles in maintaining the morphology of kidney tissue. To further confirm that actin, $\alpha$-actinin 4 , myosin, and $\alpha$-tubulin were substantially $O$-GlcNAcylated and to examine both the protein level and the level of $O$-GlcNAcylation of these proteins in the diabetic 


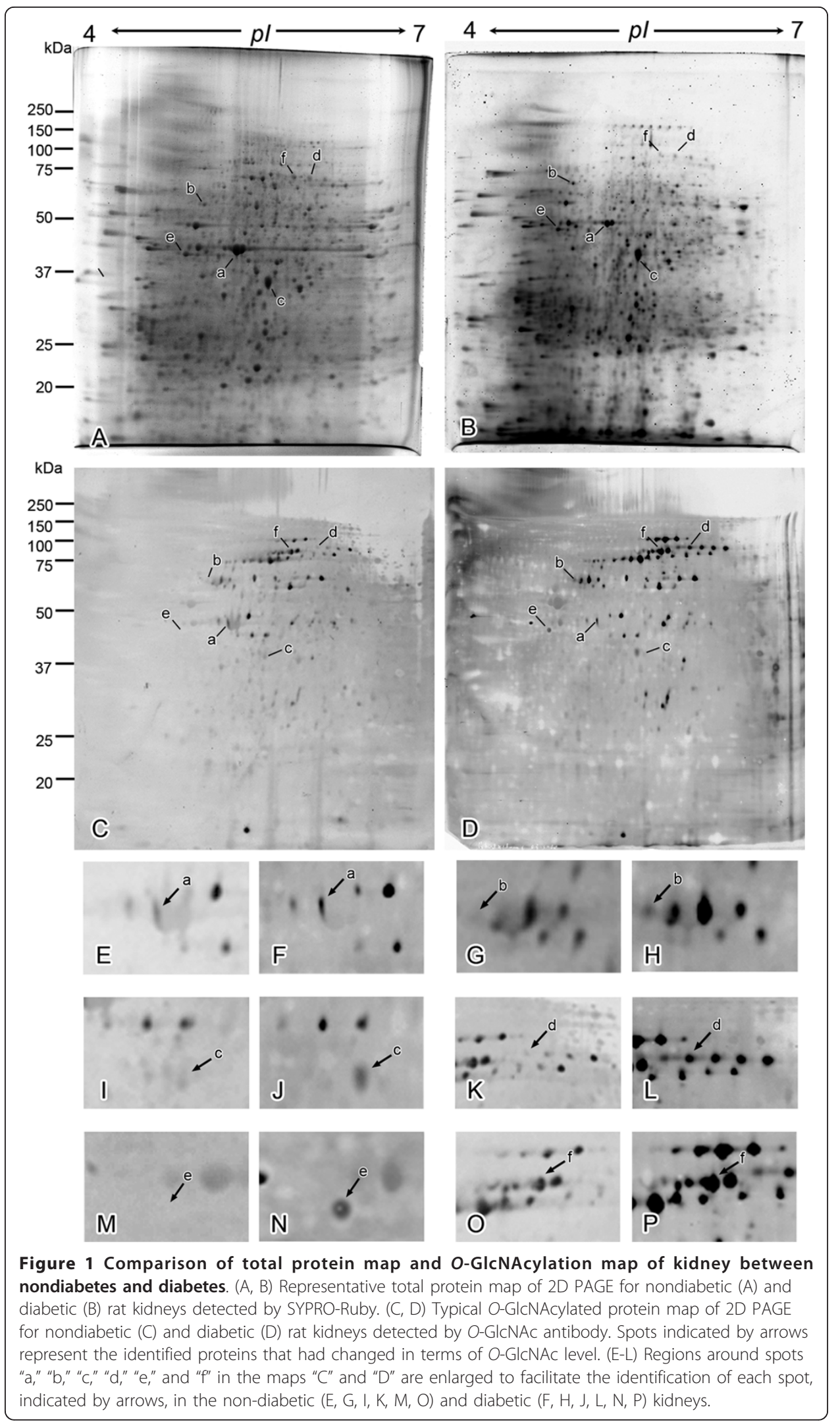


Table 1 Proteins showing an increase in the 0 -GIcNAcylation level in the diabetic kidney

\begin{tabular}{|c|c|c|c|c|c|c|c|c|}
\hline \multirow[t]{2}{*}{ Spot } & \multirow[t]{2}{*}{ Protein } & \multirow[t]{2}{*}{ Theoretical mass $(\mathrm{kDa}) / \mathrm{pl}$} & \multirow[t]{2}{*}{ Score $^{(a)}$} & \multirow{2}{*}{$\begin{array}{l}\text { Peptides } \\
\text { searched }\end{array}$} & \multirow{2}{*}{$\begin{array}{l}\text { Peptides } \\
\text { matched (a) }\end{array}$} & \multicolumn{2}{|l|}{ Fold change $^{b)}$} & \multirow{2}{*}{$\begin{array}{l}\text { Sequence } \\
\text { Coverage (\%) }\end{array}$} \\
\hline & & & & & & Wistar(control) & GK & \\
\hline $\mathrm{a}$ & Actin & $42.1 / 5.29$ & 70 & 20 & 8 & 1 & 2.15 & 22 \\
\hline b & $\alpha$-actinin 4 & 105.0/5.27 & 118 & 40 & 17 & 1 & 2.05 & 21 \\
\hline$c$ & myosin heavy chain & $222.5 / 5.69$ & 54 & 18 & 12 & 1 & 2.22 & 9 \\
\hline d & $\alpha$-tubulin & $50.6 / 4.95$ & 59 & 51 & 9 & 1 & 2.75 & 33 \\
\hline $\bar{e}$ & ATP-synthase $\beta$ & $56.3 / 5.19$ & 62 & 27 & 9 & $N D^{c)}$ & $\uparrow^{d)}$ & 23 \\
\hline$f$ & pyruvate carboxylase & $130.4 / 6.34$ & 73 & 30 & 14 & 1 & 4.32 & 16 \\
\hline
\end{tabular}

Spots a-f correspond to those shown in Figure 1.

a) Values of score and peptides matched were determined according to the Mascot search engine on the Swiss-Prot web server (Database: SwissProt 57.6).

b) Fold changes were calculated by using the mean values for the spots of diabetic GK rat kidney relative to those of normal control Wistar rat kidney, which are indicated as "1."

c) ND: not detected.

d) Up-arrow: This O-GlcNAcylated spot appeared only in the diabetic kidney. 
kidney, we performed immunoprecipitation using antibodies against these proteins and compared relative protein expression and $O$-GlcNAcylation by immunoblotting using anti-protein or anti-O-GlcNAc, respectively. As shown in Figure 2, the level of these proteins did not change except in the case of $\alpha$-actinin 4, whose level increased in the diabetic kidney. In contrast, the $O$-GlcNAcylation level of each protein from the diabetic kidney relative to that of each from the nondiabetic one was significantly higher (Figure 2). There might be other proteins in the immunoprecipitants which interacted with or bound to the target proteins. If such proteins were present, it is important to

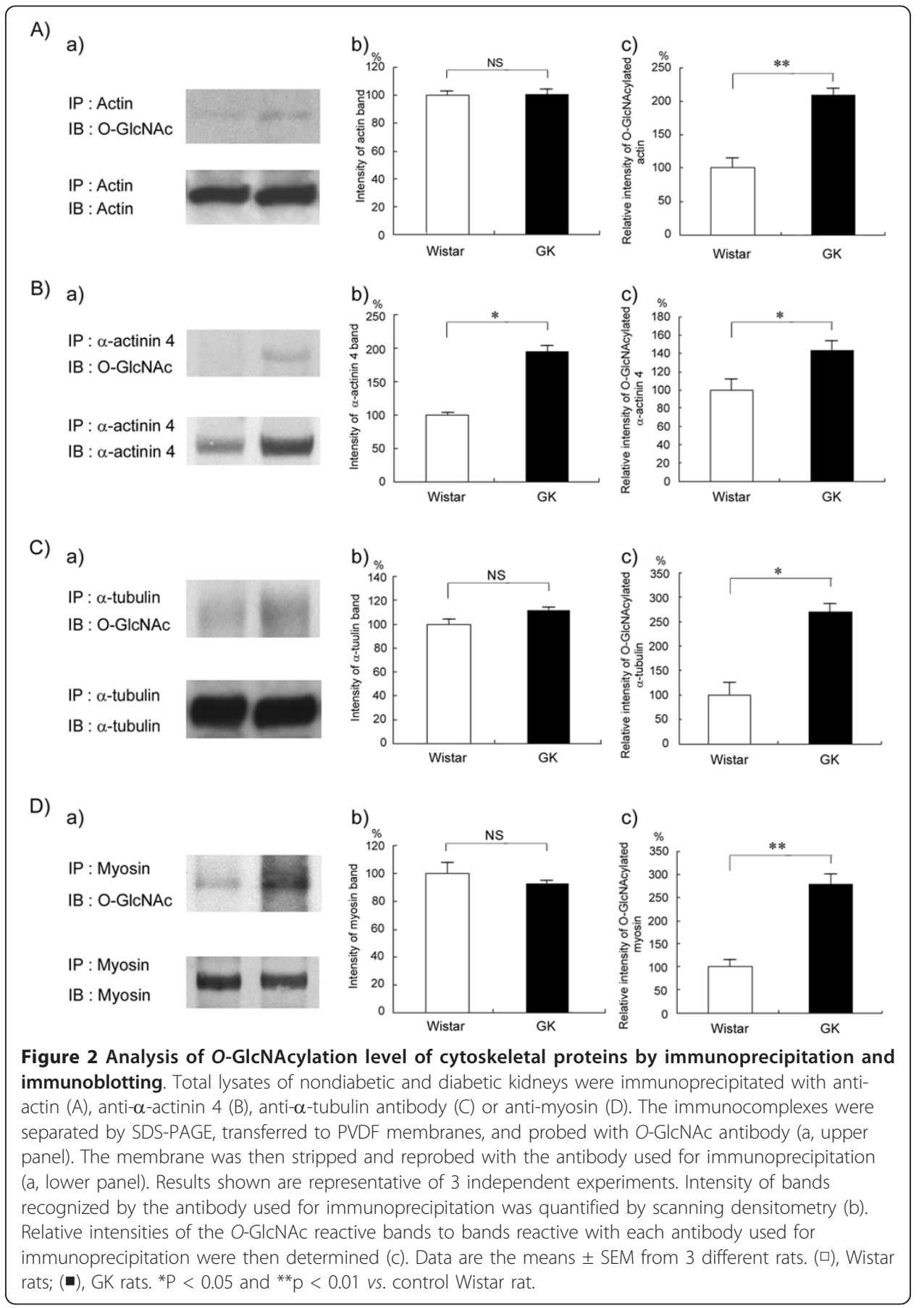


determine the expression level and the O-GlcNAcylation level of these proteins between the diabetic and nondiabetic kidney in the future.

\section{Immunohistochemical study on cytoskeletal proteins}

To examine the localization of the identified cytoskeletal proteins (actin, $\alpha$-actinin 4, $\alpha$-tubulin, and myosin), we carried out immunohistochemical analyses of the glomeruli and proximal tubules (Figure 3).

The intensity of immunoreactivity for actin in the glomerulus from the diabetic kidney was increased, especially in its mesangial cells and podocytes (Figure 3B). This result is consistent with an earlier study on the GK rat [21]. The immunoreactivity against actin was also detected in the brush border in the proximal tubules. However, its intensity did not change in the diabetic kidney (Figure 3B)

The immunofluorescence indicating $\alpha$-actinin 4 was observed as a linear pattern around the lumen of the glomerular capillary, and its intensity in the glomerulus was

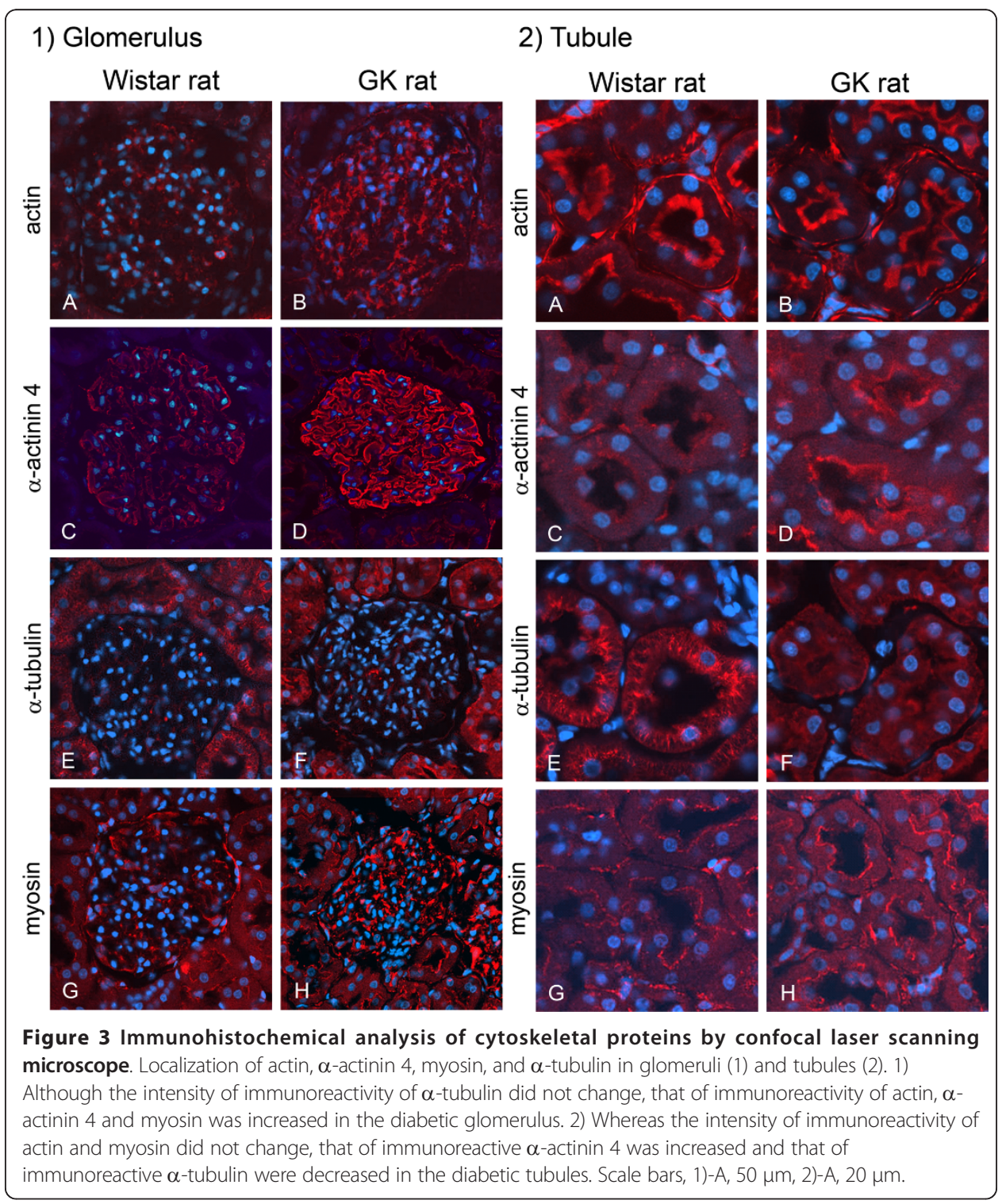


increased in the diabetic kidney (Figure 3D). $\alpha$-Actinin 4 was also localized in the brush border area at the luminal side of tubules, and the immunoreactivity was greater in the sections from the diabetic kidney (Figure 3D).

In the glomerulus the immunoreactivity of $\alpha$-tubulin was weak and did not change in the section from the diabetic kidney (Figure 3F). In the sections showing the proximal tubules of the nondiabetic kidney, a striated immunoreactivity pattern was observed; whereas a diffuse one was noted in the case of the diabetic kidney (Figure 3F).

Whereas weak immunofluorescence was observed in the glomerulus from the normal kidney (Figure 3-1G), intense immunoreactivity was observed in that from the diabetic kidney (Figure 1C-P). Myosin immunoreactivity was also observed in the brush border area of the proximal tubules in sections from both normal and diabetic kidneys, but no difference in intensity was observed between normal and diabetic proximal tubules (Figure 3H).

\section{Immuno-electron microscopy of $\alpha$-actinin 4}

Because $\alpha$-actinin 4 has been identified as the causal gene for familial focal segmental glomerulosclerosis and is considered to play an important role in the maintenance of podocyte morphology [22,23], we further examined the precise localization of $\alpha$-actinin 4 in the glomerulus and tubules by performing immuno-electron microscopy.

As we had reported previously [10], in the diabetic kidney of the GK rat thickened basement membranes of the capillaries in the glomerulus and fused foot processes of podocytes were observed (Figure 4A, B). Immuno-electron microscopy revealed that in the normal kidney the immunogold particles labeling $\alpha$-actinin 4 were localized in the cortical area of foot processes of podocytes except beneath the basal plasma membrane (Figure $4 \mathrm{C}$ ) but that the localization was different in the diabetic kidney; i.e., $\alpha$-actinin 4 was distributed not only in the cortical area but also in the inner area of foot processes (Figure 4D), and the number of immuno-gold particles was greater in the sections from the diabetic kidney (Figure 4E).

Microvilli at the luminal side of proximal tubules from the diabetic GK rats were occasionally swollen and had become bulbous, whereas those from the non-diabetic Wistar rats were regularly shaped and closely packed (Figure 5A, B). Immuno-electron microscopy of sections from the diabetic kidney revealed that colloidal gold particles labeling $\alpha$-actinin 4 were localized along the full length of the microvilli of proximal tubules of diabetic kidney, whereas in those from the normal kidney the particles tended to be localized near the bottom of the microvilli (Figure 5C, D). The colloidal gold particle density indicating $\alpha$-actinin 4 in the microvilli was increased significantly in the sections from the diabetic kidney (Figure 5E). The gold label was also observed in the adherence junctions of proximal tubule cells (Figure 5F, G), but no significant difference in the labeling density or localization of $\alpha$-actinin 4 in these junctions was observed between normal and diabetic kidney sections.

\section{In situ proximity ligation assay of O-GIcNAcylated cytoskeletal proteins}

$O$-GlcNAcylation of proteins is a common type of posttranslational modification. The in situ PLA was developed to image protein-to-protein interactions and posttranslational modifications in cells and tissues [13,14]. Using this in assay, we examined the 


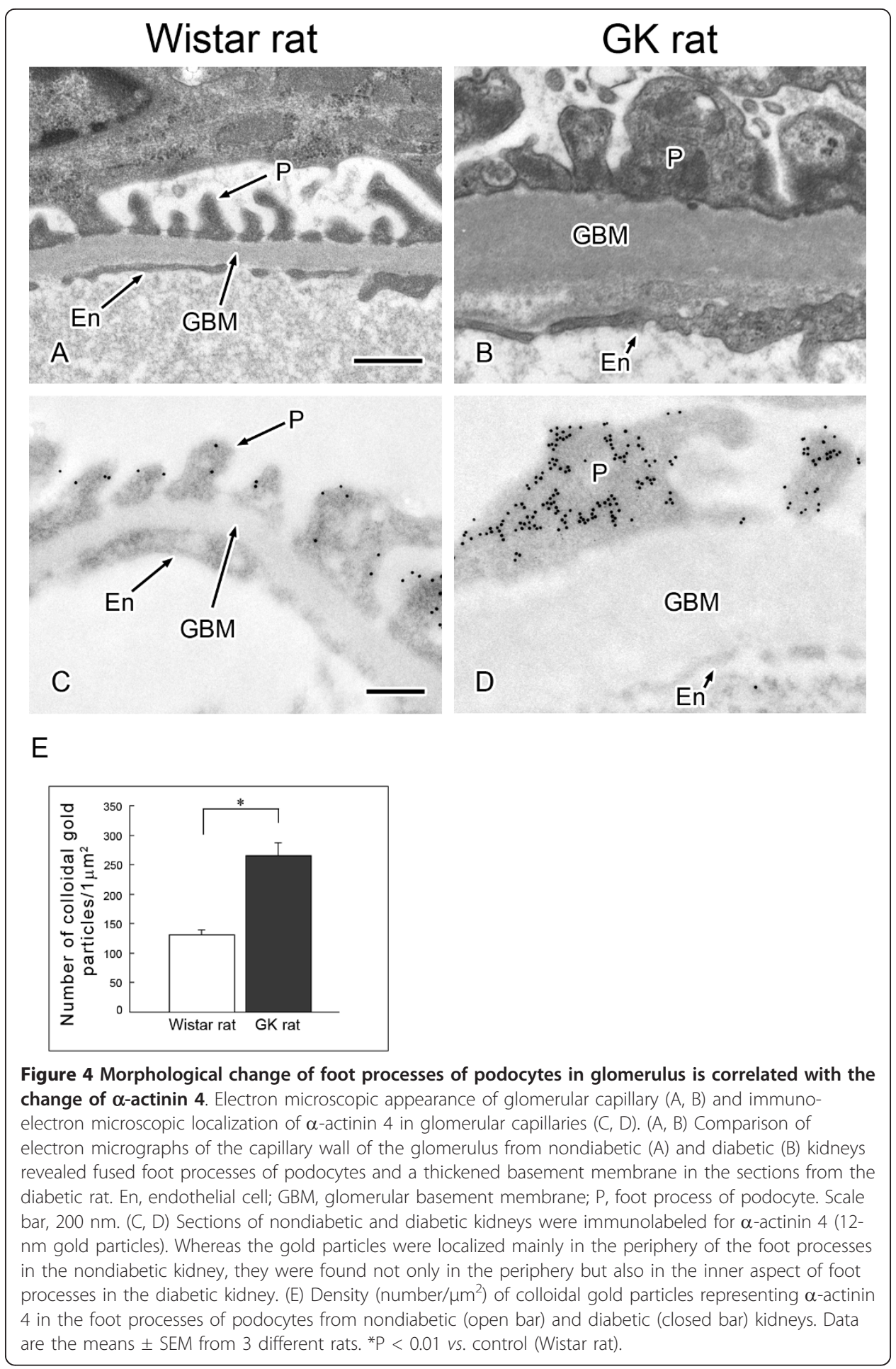

localization of $O$-GlcNAcylated cytoskeletal proteins (actin, $\alpha$-actinin $4, \alpha$-tubulin, and myosin) and quantified their signals.

Signals of $O$-GlcNAcylated-actin, $\alpha$-actinin $4, \alpha$-tubulin, and myosin were observed in the glomerulus (Figure 6-1) and tubules (Figure 6-2) in sections of normal kidney. The number of signals of all these $O$-GlcNAcylated-proteins was significantly increased 


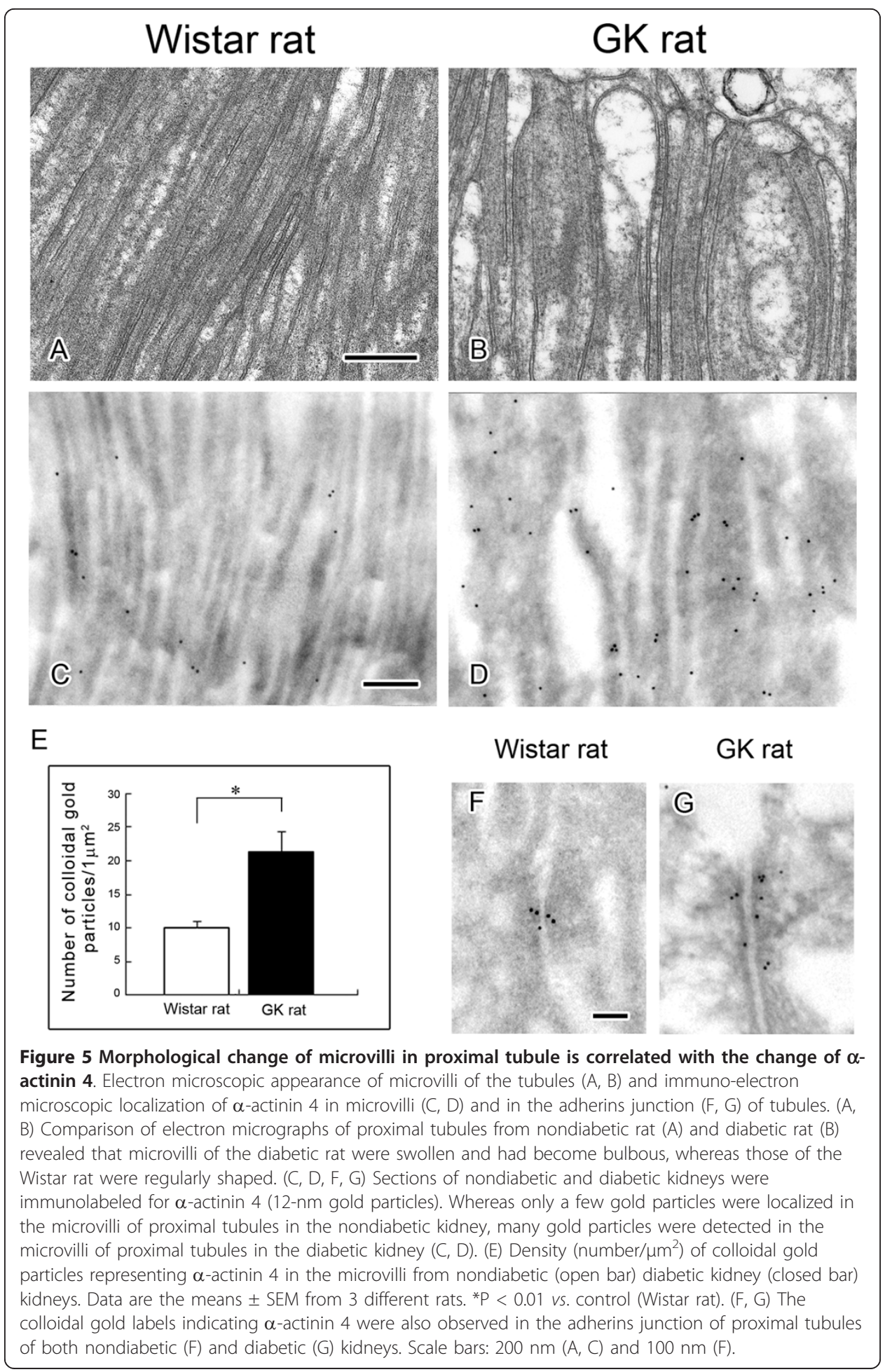

in both the glomeruli and tubules in sections of the diabetic kidney (Figure,6-1, -2). In the diabetic kidney sections, the signals of $O$-GlcNAcylated $\alpha$-actinin 4 were increased especially in the podocytes of the glomeruli (Figure 6-1D). The localizations of $O$ GlcNAcylated actin, $\alpha$-actinin 4 , and myosin shown by in situ PLA were almost the same as those observed in the conventional immunohistochemical study (Figure 3). 


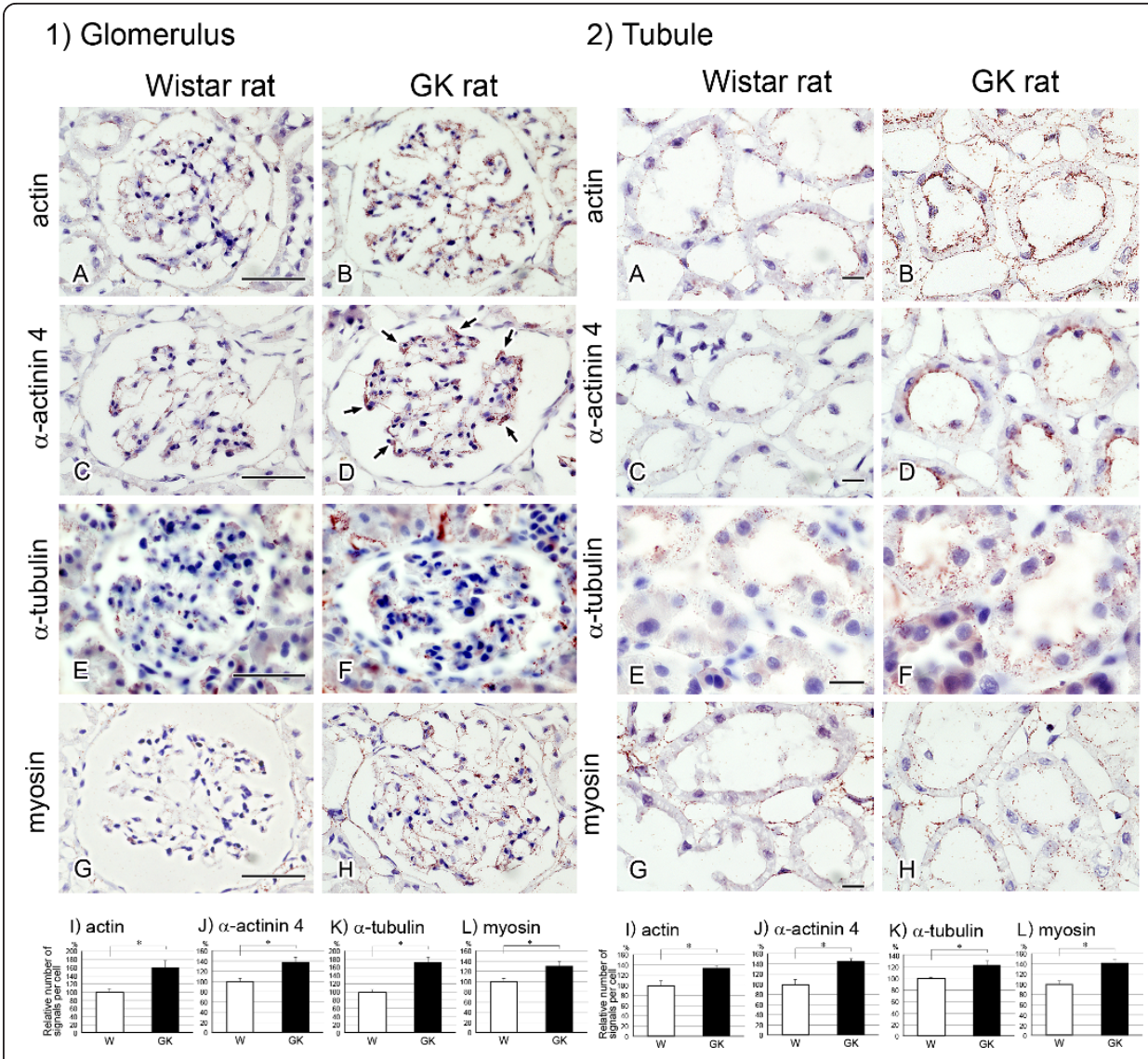

Figure 6 Analysis of O-GIcNAcylated cytoskeletal proteins by using the in situ PLA assay. Localization of O-GlcNAcylated actin (A, B), O-GlcNAcylated $\alpha$-actinin 4 (C, D), O-GlcNAcylated $\alpha$-tubulin (, , F) and $O-G$ clcNAcylated myosin $(G, H)$ in the glomerulus (1) and tubule (2) of normal $(A, C, E, G)$ and diabetic $(B, D, F, H)$ rats. Arrows in $D$ indicate podocytes. I-L) Relative number of signals per cell. Ten different glomeruli and tubules were obtained from each sample. Signals were analyzed by Blob-Finder software. Values represent means \pm SEM from 3 different rats. ${ }^{* P}<0.05$ vs. control Wistar rat (W).

However, with respect to the $O$-GlcNAcylated $\alpha$-tubulin, the striated signal pattern in the normal kidney revealed by the conventional immunohistochemistry (Figure 3-2E) was not observed (Figure 6-2E); rather, the signals were diffusely distributed in the tubules in sections of normal and diabetic kidney (Figure 3F). This observation indicates that polymerized tubulin may not be O-GlcNAcylated.

\section{Discussion}

In this study using proteomic analysis, we identified several $O$-GlcNAcylated proteins including cytoskeletal proteins, actin, $\alpha$-actinin $4, \alpha$-tubulin, and myosin and we demonstrated that their extent of $O$-GlcNAcylation was elevated in the diabetic kidney. For the first time, in situ PLA studies demonstrated the localization of O-GlcNAcylated cytoskeletal proteins and confirmed their increase in $O$-GlcNAcylation.

Actin is an important cytoskeletal protein and is O-GlcNAcylated [24]. O-GlcNAcylation of actin may modulate the actin-tropomyosin interaction and be involved in the polymerization of myosin heavy chains [24]. High glucose alters protein kinase C-dependent actin assembly in both cultured glomerular mesangial cells and podocytes [25]. Mapping of $O$-GlcNAcylation sites revealed that the $\mathrm{Ser}^{201}$ residue of actin is both $O$-GlcNAcylated 
and phosphorylated (Table 2) [26,27]. The increased O-GlcNAcylation of actin may cause the abnormal assembly of the cytoskeleton, which might have led to the morphological changes in the foot processes and microtubules in the diabetic kidney. It is important to note that if even a small percentage of actin monomers were $O$-GlcNAcylated at a key site regulating filament assembly, this could significantly alter polymerization of the actin network.

The second $O$-GlcNAcylated protein that we identified was $\alpha$-actinin 4 . This is the first report to show that $\alpha$-actinin 4 is $O$-GlcNAcylated. $\alpha$-actinin 4 is thought to play an important role in the maintenance of morphology of podocytes by cross-linking with the cortical actin network, which serves as an anchor for a variety of intracellular structures [28,29]. $\alpha$-actinin 4 is also involved in the bundling of F-actin and vesicular trafficking and has also been identified as the causal gene for familial focal segmental glomerulosclerosis [22]. The present results suggest that abnormal O-GlcNAcylation of $\alpha$-actinin 4 and actin may affect the crosslinking to actin and cause the morphological changes seen in the podocyte foot processes in the glomeruli and in the microvilli of tubules. $\alpha$-Actinin 4 is reported to be phosphorylated at its $\mathrm{Tyr} / \mathrm{Ser} / \mathrm{Thr}$ residues $[27,30]$, but the $O$-GlcNAcylation sites have not been reported yet. The predicted $O$ GlcNAcylation sites $\operatorname{Ser}^{262}$ and $\operatorname{Ser}^{269}$ are located near the phosphorylation site $\mathrm{Tyr}^{265}$, where the phosphorylation at this site regulates the interaction of $\alpha$-actinin 4 with actin and alters its intracellular location and conformation (Table 2) [31]. Further study remains to be done to clarify the precise $O$-GlcNAcylation sites of $\alpha$-actinin 4 and their role in the normal and diabetic kidneys.

$\alpha$-Tubulin is a component of microtubules, which are involved in reabsorption of substances in kidney tubules via the transport of vesicles from the luminal surface to the basal surface of the tubules. It was reported that $O$-GlcNAcylation of miro and milton (OIP106 and GRIF-1) plays a crucial role in the transportation of vesicles and mitochondria via microtubules [32]. The present in situ PLA study showed that the $O$ GlcNAcylation of $\alpha$-tubulin was increased in both the glomerulus and tubule. It has been reported that $\alpha$-tubulin is $O$-GlcNAcylated [33] and phosphorylated [34-36], but the $O$-GlcNAcylation sites in it have not been determined yet. $\mathrm{Thr}^{271}$ residue, one of the predicted $O$-GlcNAcylation sites, is located next to the phosphorylation sites $\mathrm{Tyr}^{272}$ (Table 2). By inhibiting the phosphorylation of $\mathrm{Tyr}^{272}$, O-GlcNAcylation of $\mathrm{Thr}^{271}$ may regulate microtubule formation and/or interaction with ligands, such as tau, microtubule-associated proteins 1, 2 (MAPs-1, 2), which are also O-GlcNAcylated.

Table 2 Comparison between phosphorylation sites and O-GIcNAcylation sites

\begin{tabular}{|c|c|c|}
\hline Protein & Phosphorylation sites & O-GlcNAcylation sites \\
\hline actin & S35, S201 27 & S54, S157, S201, S234, S37026 \\
\hline$\alpha$-actinin 4 & Y265, S423, S621, K625, T66727, 30 & S262, S269, T612, S613, S656* \\
\hline$\alpha$-tubulin & Y103, Y224, S232, Y272, T334 35,36 & S6, S158, S178, T271, S419** \\
\hline $\begin{array}{l}\text { myosin } \\
\text { heavy } \\
\text { chain }\end{array}$ & $\begin{array}{l}\text { Y389, Y410, S949, S1038, Y1375 } \\
\text { Y1415, Y1852, S1956, T1960 } 39,40\end{array}$ & $\begin{array}{l}\text { S172, S179, S196, S392, S622, S626, S643, S644, S749, S880, } \\
\text { S1038, S1102, S1148, S1159, S1189, S1200, S1299, S1308, } \\
\text { S1336, S1470, S1471, S1596, S1597, S1600, S1606, S1710, } \\
\text { S1711, S1777, S191626 }\end{array}$ \\
\hline
\end{tabular}

Boldface type indicates the site at which O-GIcNAcylation and O-phosphorylation compete or their respective sites that are close to each other.

* Predicted by YinOYang1.2 prediction server http://www.cbs.dtu.dk/services/YinOYang/

* * Predicted by dbOGAP prediction server https://cbsb.lombardi.georgetown.edu/OGAP.html 
Recently it was demonstrated that $O$-GlcNAcylation of tubulin inhibits its polymerization and negatively regulates microtubule formation [37]. The present results suggest that the reabsorption of certain substances in the proximal tubules by transportation via microtubules may be hampered as polymerization of tubulin is inhibited by its abnormal O-GlcNAcylation.

Myosin is another important cytoskeletal protein, and it is also O-GlcNAcylated $[24,38]$. In situ PLA revealed that the O-GlcNAcylation of myosin was increased in both glomeruli and tubules, where myosin plays an important role in the maintenance of the morphology of glomerular cells and microvilli of tubules. Mapping of O-GlcNAcylation sites revealed that $\operatorname{Ser}^{1038}$ residue is $O$-GlcNAcylated (Table 2) [26] and this same residue has also been shown to be phosphorylated (Table 2) $[39,40]$. The role of $O$-GlcNAcylation and phosphorylation of $\mathrm{Ser}^{1038}$ residue remains to be clarified.

In conclusion these results suggest that in the diabetic kidney the morphological changes in the glomerulus and tubules may be ascribed to the abnormal O-GlcNAcylation of cytoskeletal proteins including $\alpha$-actinin 4 , which $O$-GlcNAcylation is induced by hyperglycemia-enhanced flux through the hexosamine biosynthetic pathway. $\alpha$-Actinin 4 will be a good maker to examine the relation between $O$-GlcNAcylation and diabetic nephropathy. In situ PLA method could be used for the clinical diagnosis to localize the $O$-GlcNAcylated proteins and quantify them when the antibody against $O$-GlcNAcylated protein is not available. Further studies need to be carried out to clarify the roles of $O$-GlcNAcylation of cytoskeletal proteins in the maintenance of cell morphology and the relationships between $O$-GlcNAcylation of proteins and the etiology of diabetes complications by the glycomic approaches [41].

\section{List of Abbreviations}

O-GlcNAc: O-linked N-acetylglucosamine; O-GlcNAcylation: modification of proteins by O-GICNAC; PLA: in situ proximity ligation assay; GK rat: Goto-Kakizaki rat; OGT: O-GICNAc transferase; MS: Mass Spectrometry; LAB solution: liberate antibody binding solution; TBS-T: Tris-buffered saline-0.1\% Tween 20; MAPs-1, 2: microtubule-associated proteins 1, 2.

\section{Acknowledgements}

The authors thank Ms. Sachie Matubara, Ms. Miki Kanai, Ms. Tomoko Miura and Ms. Sayuri Koroishi (Laboratory for Electron Microscopy and Department of Anatomy, Kyorin University School of Medicine) for technical assistance. This study was supported in part by Grant-in-Aid for Scientific Research from the Japanese Ministry of Education, Culture, Sports, Science and Technology (C-20590198 to YA), from Japan Diabetes Foundation (to YA), from the Kazato Research Foundation (to YA), from Kyorin University School of Medicine, Kyorin Medical Research Award 2010 (to YA) and by NIH R01 DK61671 (to GWH).

\footnotetext{
Author details

1Department of Anatomy, Kyorin University School of Medicine, Mitaka, Tokyo 181-8611, Japan. ${ }^{2}$ Research Team for Mechanism of Aging, Tokyo Metropolitan Institute of Gerontology, Itabashi, Tokyo 173-0015, Japan. ${ }^{3}$ Complex Carbohydrate Research Center, University of Georgia, Athens, GA 30602, USA. ${ }^{4}$ Department of Chemistry, University of Georgia, Athens, GA 30602, USA. ${ }^{5}$ Department of Biochemistry and Molecular Biology, University of Georgia, Athens, GA 30602, USA. ${ }^{6}$ Department of Biological Chemistry, Johns Hopkins University School of Medicine, Baltimore, MD 21205, USA.
}

\section{Authors' contributions \\ YA performed all experiments, contributed to discussion, and drafted the manuscript. YM, TT and TE performed MS, analyzed data, contributed to discussion and edited the manuscript. HK contributed to discussion and edited the manuscript. MAW, LW, GJB contributed to antibodies and discussion. GWH contributed to discussion, and reviewed and edited the manuscript. All authors read and approved the final manuscript.}

\section{Conflicts of interests}

The authors declare that they have no competing interests. 


\section{References}

1. Wells L, Vosseller K, Hart GW: Glycosylation of nucleocytoplasmic proteins: signal transduction and O-GIcNAc. Science 2001, 291:2376-2378.

2. Hart GW, Housley MP, Slawson C: Cycling of O-linked $\beta$-N-acetylglucosamine on nucleocytoplasmic proteins. Nature 2007, 446:1017-1022.

3. Butkinaree $\mathrm{C}$, Park K, Hart GW: O-linked $\beta$-N-acetylglucosamine (O-GIcNAc): Extensive crosstalk with phosphorylation to regulate signaling and transcription in response to nutrients and stress. Biochim Biophys Acta 2010, 1800:96-106.

4. Yang $X$, Ongusaha PP, Miles PD, et al: Phosphoinositide signaling links O-GIcNAc transferase to insulin resistance. Nature 2008, 451:964-969

5. Teo CF, Wollaston-Hayden EE, Wells L: Hexosamine flux, the O-GICNAc modification, and the development of insulin resistance in adipocytes. Mol Cell Endocrinol 2010, 318:44-53.

6. Daniels MC, Kansal P, Smith TM, Paterson AJ, Kudlow JE, McClain DA: Glucose regulation of transforming growth factor-alpha expression is mediated by products of the hexosamine biosynthesis pathway. Mol Endocrinol 1993, 7:1041-1048.

7. Kolm-Litty V, Sauer U, Nerlich A, Lehmann R, Schleicher ED: High glucose-induced transforming growth factor $\beta 1$ production is mediated by the hexosamine pathway in porcine glomerular mesangial cells. J Clin Invest 1998, 101:160-169.

8. Goldberg HJ, Scholey J, Fantus IG: Glucosamine activates the plasminogen activator inhibitor 1 gene promoter through Sp1 DNA binding sites in glomerular mesangial cells. Diabetes 2000, 49:863-871.

9. Weigert C, Brodbeck K, Sawadogo M, Häring HU, Schleicher ED: Upstream stimulatory factor (USF) proteins induce human TGF- $\beta 1$ gene activation via the glucose-response element-1013/-1002 in mesangial cells: up-regulation of USF activity by the hexosamine biosynthetic pathway. J Biol Chem 2004, 279:15908-15915.

10. Akimoto $\mathrm{Y}$, Yamamoto $\mathrm{K}$, Munetomo $\mathrm{E}$, et al: Elevated post-translational modification of proteins by $\mathrm{O}$-linked $\mathrm{N}$ acetylglucosamine in various tissues of diabetic Goto-Kakizaki rats accompanied by diabetic complications. Acta Histochem Cytochem 2005, 38:131-142.

11. Akimoto $Y$, Hart GW, Wells $L$, et al: Elevation of the post-translational modification of proteins by $\mathrm{O}$-linked $\mathrm{N}$ acetylglucosamine leads to deterioration of the glucose-stimulated insulin secretion in the pancreas of diabetic Goto-Kakizaki rats. Glycobiology 2007, 17:127-140.

12. Degrell P, Cseh J, Mohás M, et al: Evidence of O-linked N-acetylglucosamine in diabetic nephropathy. Life Sci 2009, 84:389-393.

13. Fredriksson S, Gullberg M, Jarvius J, et al: Protein detection using proximity-dependent DNA ligation assays. Nat Biotechnol 2002, 20:473-477.

14. Söderberg $\mathrm{O}$, Gullberg $\mathrm{M}$, Jarvius $\mathrm{M}$, et al: Direct observation of individual endogenous protein complexes in situ by proximity ligation. Nat Methods 2006, 3:995-1000.

15. Comer Fl, Vosseller K, Wells L, Accavitti MA, Hart GW: Characterization of a mouse monoclonal antibody specific for O-linked N-acetylglucosamine. Anal Biochem 2001, 293:169-177.

16. Teo CF, Ingale S, Wolfert MA, et al: Glycopeptide-specific monoclonal antibodies suggest new roles for O-GIcNAc. Nat Chem Biol 2010, 6:338-343.

17. Miura $\mathrm{Y}$, Kano M, Yamada $\mathrm{M}$, et al: Proteomic study on X-irradiation-responsive proteins and ageing: search for responsible proteins for radiation adaptive response. J Biochem 2007, 142:145-155.

18. Toda T, Kimura N: Standardization of protocol for immobiline 2-D PAGE and construction of 2-D PAGE protein database on world wide web home page. Jpn J Electroph 1997, 41:13-19.

19. Toda T, Kaji K, Kimura N: TMIG-2DPAGE: a new concept of two-dimensional gel protein database for research on aging. Electrophoresis 1998, 19:344-348.

20. Zieba A, Wählby $C$, Hielm F, et al: Bright-field microscopy visualization of proteins and protein complexes by in situ proximity ligation with peroxidase detection. Clin Chem 2010, 56:99-110.

21. Janssen U, Riley SG, Vassiliadou A, Floege J, et al: Hypertension superimposed on type II diabetes in Goto Kakizaki rats induces progressive nephropathy. Kidney Int 2003, 63:2162-2170.

22. Kaplan JM, Kim SH, North KN, et al: Mutations in ACTN4, encoding a-actinin-4, cause familial focal segmental glomerulosclerosis. Nat Genet 2000, 24:251-256.

23. Kos CH, Le TC, Sinha S, et al: Mice deficient in a-actinin-4 have severe glomerular disease. J Clin Invest 2003, 111:1683-1690

24. Hédou J, Bastide B, Page A, Michalski J-C, Morelle W: Mapping of O-linked $\beta$ - $N$-acetylglucosamine modification sites in key contractile proteins of rat skeletal muscle. Proteomics 2009, 9:2139-2148.

25. Zhou X, Hurst RD, Templeton D, Whiteside Cl: High glucose alters actin assembly in glomerular mesangial and epithelial cells. Lab Invest 1995, 73:372-383.

26. Ramirez-Correa GA, Jin W, Wang Z, et al: O-linked GIcNAc modification of cardiac myofilament proteins: a novel regulator of myocardial contraction function. Circ Res 2008, 103:1354-1358.

27. Huang S-Y, Tsai M-L, Chen G-Y, Wu C-J, Chen SH: A systematic MS-based approach for identifying in vitro substrates of PKA and PKG in rat uteri. J Proteome Res 2007, 6:2674-2684.

28. Nabet B, Tsai A, Tobias JW, Carstens RP: Identification of a putative network of actin-associated cytoskeletal proteins in glomerular podocytes defined by co-purified mRNAs. PLOS ONE 2009, 4:e6491.

29. Ichimura K, Kurihara H, Sakai T: Actin filament organization of foot processes in rat podocytes. J Histochem Cytochem 2003, 51:1589-1600

30. Chen $Y$, Choong L-Y, Lin $Q$, et al: Differential expression of novel tyrosine kinase substrates during breast cancer development. Mol Cell Proteomics 2007, 6:2072-2087.

31. Shao $\mathrm{H}, \mathrm{Wu} \mathrm{C}$, Wells $\mathrm{A}$ : Phosphorylation of a-actinin 4 upon epidermal growth factor exposure regulates its interaction with actin. J Biol Chem 2010, 285:2591-2600.

32. Glater EE, Megeath $\mathrm{L}$, Stowers RS, Schwarz TL: Axonal transport of mitochondria requires milton to recruit kinesin heavy chain and is light chain independent. J Cell Biol 2006, 173:545-557.

33. Walgren JLE, Vincent TS, Schey KL, Buse MG: High glucose and insulin promote O-GIcNAc modification of proteins, including a-tubulin. Am J Physiol Endocrinol Metab 2003, 284:E424-E434. 
34. Nogales E: Structural insights into microtubule function. Annu Rev Biochem 2000, 69:277-302.

35. Jørgensen C, Sherman A, Chen Gl, et al: Cell-specific information processing in segregating populations of Eph receptor ephrin-expressing cells. Science 2009, 326:1502-1509.

36. Ballif BA, Carey GR, Sunyaev SR, Gygi SP: Large-scale identification and evolution indexing of tyrosine phosphorylation sites from murine brain. J Proteome Res 2008, 7:311-318.

37. Ji S, Kang JG, Park SY, Lee J, Oh YJ, Cho JW: O-GIcNAcylation of tubulin inhibits its polymerization. Amino Acids 2011, 40:809-818.

38. Cieniewski-Bernard C, Bastide B, Lefebvre T, Lemoine J, Mounier Y, Michalski JC: Identification of O-linked Nacetylglucosamine proteins in rat skeletal muscle using two-dimensional gel electrophoresis and mass spectrometry. Mol Cell Proteomics 2004, 3:577-585.

39. Molina H, Horn DM, Tang N, Mathivanan S, Pandey A: Global proteomic profiling of phosphopeptides using electron transfer dissociation tandem mass spectrometry. Proc Natl Acad Sci USA 2007, 104:2199-2204.

40. Zanivan S, Gnad F, Wickström SA, et al: Solid tumor proteome and phosphoproteome analysis by high resolution mass spectrometry. J Proteome Res 2008, 7:5314-5326.

41. Wang Z, Hart GW: Glycomic approaches to study GlcNAcylation: Protein identification, site-mapping, and sitespecific O-GlcNAc quantitation. Clin Proteom 2008, 4:5-13.

doi:10.1186/1559-0275-8-15

Cite this article as: Akimoto et al:: Morphological changes in diabetic kidney are associated with increased $O$ GlcNAcylation of cytoskeletal proteins including $\alpha$-actinin 4. Clinical Proteomics 2011 8:15.

\section{Submit your next manuscript to BioMed Central} and take full advantage of:

- Convenient online submission

- Thorough peer review

- No space constraints or color figure charges

- Immediate publication on acceptance

- Inclusion in PubMed, CAS, Scopus and Google Scholar

- Research which is freely available for redistribution 